- Part of the role of the practitioner is to guide patients through the considerable information on their condition available through the Internet, and to aid in arriving CPD PAPER at a favourable treatment plan.

- An overview of treatment approaches, including the use of botulinum toxin, has been discussed.

- Ultrasonograpy in conjunction with directed blood tests is a useful aid to diagnosis of hypertrophy.

- Hypertrophy may affect any of the masticatory muscles in any combination.

\title{
Variable presentation of temporalis hypertrophy - A case report with literature review
}

\author{
S. Rokadiya ${ }^{1}$ and N. J. Malden ${ }^{2}$
}

\begin{abstract}
A case of painful, initially bilateral, and then unilateral temporalis hypertrophy in a 33-year-old Caucasian woman is presented in a primary care setting; the aetiology of the hypertrophy was considered reactive in nature. Medical and supportive treatment was successful in providing symptomatic relief. Further treatment including an intramuscular botulinum injection was considered by the patient with a view to reduction of the chronic enlargement of her temporalis and masseter musculature. A review of the literature and previous treatment regimes is also presented.
\end{abstract}

\section{INTRODUCTION}

In 1880, Legg ${ }^{1}$ described generalised hypertrophy of the masticatory muscles. This condition presented in a 10-year-old girl, with no associated pain, and with observation only the muscles remained approximately constant in size over the review period. Subsequently a number of other cases of generalised masticatory muscle hypertrophy have been reported ${ }^{2,3}$ but cases of isolated temporalis hypertrophy have remained low. ${ }^{4,5}$ Generalised masticatory muscle hypertrophy may affect the temporalis muscles, masseters and medial pterygoids in a variety of combinations.

Controversy surrounds the aetiology of masticatory muscle hypertrophy and the condition has been divided into acquired and congenital forms. ${ }^{4}$ It need not be parafunctional or dysfunctional in origin and possible causes include muscle

\footnotetext{
${ }^{1 *}$ Oral Surgery Department, Edinburgh Dental Institute, Lauriston Building, Lauriston Place, Edinburgh, EH3 9HA ${ }^{2}$ Associate Specialist in the Department of Oral Surgery, Edinburgh Dental Institute, 4th Floor, Lauriston Building, Lauriston Place, Edinburgh, EH3 9HA

${ }^{*}$ Correspondence to: Dr Suhail Rokadiya

Email:suhail.rokadiya@gmail.com
}

\section{Refereed Paper}

Accepted 8 March 2006

DOI: $10.1038 /$ sj.bdj.4813850

๑) British Dental Journal 2006; 201: 153-155 hyperactivity, mandibular retrognathia or an imbalance in the extra-pyramidal neurotransmitter. ${ }^{6}$ However, when there is a case of isolated temporalis hypertrophy, more sinister aetiologies should be considered such as idiopathic inflammatory myopathy, rhabdomyosarcoma and infiltrative leukaemias and lymphomas. ${ }^{6}$

\section{CASE REPORT}

A 33-year-old Caucasian female was referred to the oral surgery department by her GP complaining of acutely painful swelling of both temporal regions. She described her pain as like having a vice clamped around the head. The swelling had a three day history on presentation but there had been a similar episode reported five years previously. On that occasion the swelling had spontaneously gone into remission. For the more recent episode the swellings were felt to be most pronounced on the day they had arisen, but had slightly subsided on presentation to the clinic. There was a history of parafunction in that the patient claimed to "bang her teeth together'. The patient also considered herself to be experiencing a stressful period in her life, as was the case on the previous occasion.

The medical history included moderate to severe asthma, a history of coeliac disease which was responding slowly to dietary modifications, and a slight mitral and tricuspid valve regurgitation. Medication at the time of presentation included salbutamol sulphate (Ventolin), fluticasone propionate (Seretide), zafirlukast (Accolate) and budesonide (Symbicort) for the treatment of moderate to severe asthma, also gabapentin $800 \mathrm{mg}$ and occasional use of ibuprofen for the symptomatic relief of persistent neuroma pain around the knee.

On examination there were obvious acutely tender swellings occupying the sites of both temporalis muscles (Fig. 1). The temporalis enlargement hardened on clenching and this was associated with increased pain. Ultrasonography demonstrated a homogenous, soft tissue enlargement of both temporal muscle regions consistent with muscle hypertrophy but was otherwise unremarkable. Over the counter analgesics were not considered to be helping her symptoms.

Differential diagnosis at this time included reactive temporalis hypertrophy, acute myositis, fibromyalgia, systemic lupus erythematosis, primary biliary cirrhosis, systemic sclerosis and infiltrative leukaemia. It was of note in this case that the patient had performed an extensive search of the internet and had developed an opinion on possible diagnoses and management strategies. 


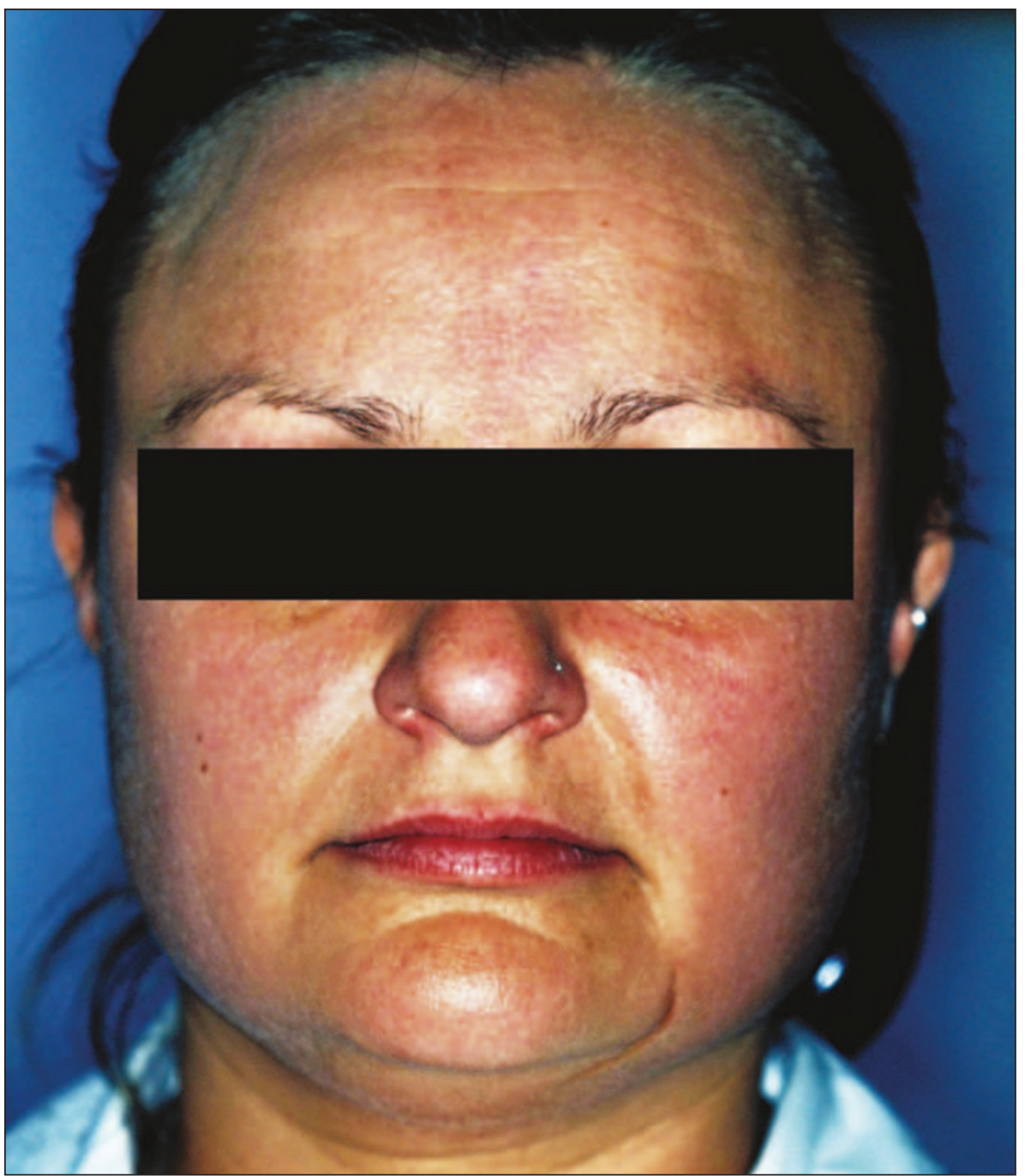

Fig. 1 Bilateral Temporalis Hypertrophy

Initial treatment included the prescription of amitriptyline hydrochloride - a tricyclic antidepressant effective in relieving muscle pain - in increasing doses, up to $150 \mathrm{mg}$ nocte. This exhibited a doserelated relief. The patient kept a record of pain-free days, which proved a helpful means of monitoring the course of the condition. A lower soft bite-freeing splint was constructed and the patient reported some added relief while wearing this. Electromyotherapy, effectively an electrical massage, was unsuccessful. Further investigations included haematological tests including full blood count, urea and electrolytes to assess kidney function as well as liver function tests which were all normal or negative. Blood tests to discount autoimmune diseases all returned negative and included anti-parietal cell antibodies (Abs), anti-mitochondrial Abs, anti-smooth muscle Abs and anti-nuclear Abs.

Following a period of just over three months with reduced pain, the patient presented again, this time with a unilateral painful enlargement of her left temporalis. Upon review of her medication the patient volunteered the information that she had emotionally traumatic aetiology. ${ }^{4}$ Biopsy of the temporalis muscle was suggested as a tool for definitive diagnosis, ${ }^{4,6}$ and use of a full blood count and erythrocyte sedimentation rate blood tests, as well as computerised tomography (CT) or magnetic resonance imaging (MRI) for exclusion of any underlying pathology. ${ }^{7}$ While radiationfree MRI is a more useful imaging tool than the relatively inexpensive CT, ultrasonography - which is cheaper, radiation-free and demonstrates the homogeneity and relative size of soft tissues - was considered worthwhile in this case. Had any concerns been raised further qualitative scanning modalities would have been pursued.

Histologically, hypertrophy can be divided into reactive and non-reactive forms. Reactive hypertrophy is due to an increased muscle workload and affects masticatory muscle through progressive change of the Type 2 fibres to the greater diameter Type I fibre. In contrast, the muscles of the limbs undergoing an increased workload increase the oxidative metabolic capacity of existing Type I fibres. Thus, biopsy of suspected reactive hypertrophied masticatory muscle will show this change. ${ }^{2}$

As a consequence of the history of parafunction, previous temporalis enlargement and absence of pathology from the haematology tests, a diagnosis of temporalis hypertrophy was made. There was no MRI, CT scan or biopsy for histological analysis performed in this case as they were considered of no additional benefit to the patient.

There has been a variety of treatment regimens described for temporalis hypertrophy. Conservative treatment has traditionally been employed, ${ }^{4,7}$ with the provision of bite-freeing splints as an adjunct to an increased awareness of parafunction, when present. ${ }^{8}$

Medical management has been restricted to the prescription of systemic muscle relaxants. ${ }^{7}$ Although there is an occasional side effect of xerostomia from amitriptyline, it is considered of therapeutic value due to its muscle relaxant activity.

Serrat et al..$^{9}$ employed gross dissective surgery under general anaesthesia for reduction of the temporalis muscle, primarily for aesthetic concerns in a 15-yearold. Morselli describes a less traumatic procedure involving 'shaving' portions of the muscle using a closed technique with local anaesthetic and sedation only. ${ }^{10}$

The relative morbidity and cost involved with surgery has seen a recent trend towards the comparatively non-invasive use of botulinum toxin, administered by local infiltration into the muscle. This well known toxin has been widely used for the treatment of a variety of conditions 
including masseteric hypertrophy. Isaac first demonstrated its efficacy in a patient with painless unilateral temporalis hypertrophy. ${ }^{5}$ In this case the treatment was considered successful at 12 months post treatment. Treatment with botulinum toxin has been shown to improve the quality of life by an average of five points (assessed using the Visual Analogue Scale) in patients suffering from masticatory muscle hypertrophy, although this study primarily involved patients with masseteric hypertrophy. Furthermore, Kim et al. ${ }^{6}$ reported a decrease in pain symptoms and trismus and a reduction in masseteric muscle volume of 35\% when analysed by CT mapping. The peak effect was found to be at 12 weeks, which coincided with time of maximum patient satisfaction. This study also showed a dose response to botulinum toxin.

The mechanism of action of botulinum is through cholinergic chemodenervation of motor end plates and inhibiting the gamma motor endings in muscle spindles. There is also a suggestion that botulinum toxin may have a role in pain relief directly through one of its metabolites. ${ }^{12}$ Tolerance to botulinum A toxin (due to antibody production) may be managed by switching to botulinum type $\mathrm{F}$ as an alternative. However, the duration of action of botulinum $\mathrm{F}$ is shorter than botulinum $\mathrm{A}, 5$ so the need for repeat doses is increased. Multiple injections over a 12 month period have been reported, ${ }^{6}$ with no long lasting side effects from the toxin when used in the temporalis region.

In light of the short term effect of botulinum toxin there has been a proposal that with precise knowledge of the anatomy of the nerve to temporalis it would be possible to carry out closed selective electrical denervation. Hwang et al., ${ }^{13}$ through work on cadavers, hypothesised that hypertrophy of the antero-central part of temporalis can be reduced without disturbance to the masticatory function if the anterior division of the deep temporal nerve is selectively severed.

\section{CONCLUSION}

This case was unusual not only because of the sites of reactive muscle hypertrophy but also because of the intensity of pain reported. Working in partnership with the patient's medical practitioner, a regime of medical and conservative management resulted in a remission of painful symptoms.

Patients using the Internet to obtain information on clinical problems may receive unbalanced information, and initially this particular patient was sceptical about reports of the use of botulinum or surgery for her condition as posted on the internet. Following a further search by the authors a more balanced selection of publications was made available to the patient. This helped her make a more informed choice about her future treatment options and indeed she has decided to undergo treatment with botulinum toxin.
The authors would like to thank Joan Patterson for her secretarial support and Dr John Gibson and Miss Gemma Dalzell for their helpful advice.

1. Legg J W. Enlargement of the temporal and masseter muscles on both sides. Trans Patho/ Soc Lond 1880; 31:361-366.

2. Harriman D G. The histochemistry of reactive masticatory muscle hypertrophy. Muscle Nerve 1996; 19: 1447-1456.

3. Gniadecka A M, Weismann K, Herning M. Swelling of the temporal region: a case of benign masticatory muscle hypertrophy. Br J Dermatol 1997; 136: 242-244.

4. Wilson PS, Brown M S. Unilateral temporalis muscle hypertrophy: case report. Int J Oral Maxillofac.Surg 1990; 19: 287-288.

5. Isaac A M. Unilateral temporalis muscle hypertrophy managed with botulinum toxin type A. Br J Oral Maxillofac Surg 2000; 38: 571-572.

6. von Lindern J J, Niederhagen B, Appel T et al. Type A botulinum toxin for the treatment of hypertrophy of the masseter and temporal muscles: An alternative treatment. Plast Reconstr Surg 2001; 107: 327-332.

7. Lowry TR, Helling E. Unilateral temporal muscle hypertrophy: A rare clinical entity. ENT: Ear Nose and Throat J 2003; 82: 198-200.

8. Kalish G H, Gellis SS. Hypertrophy of the masseter or temporalis muscles or both. Am J Dis Child 1971; 121: 346-347.

9. Serrat A, Garcia-Cantera J M, Redondo L M. Isolated inilateral temporalis muscle hypertrophy: a case report. Int J Oral Maxillofac Surg 1998; 27: 92-93.

10. Morselli P G. Temporalis muscle hypertrophy; a new plastic surgery procedure. Plast Reconstr Surg 2000; 106: 1156-1161.

11. Kim H J, Yum K W, Lee S S et al. Effects of Botulinum Toxin Type A on Bilateral Masseteric Hypertrophy evaluated with computed tomographic measurement. Dermato/Surg 2003; 29: 484-489.

12. Guyer B M. Mechanism of Botulinum Toxin in the relief of chronic pain. Current Pain and Headache Reports 1999; 3: 427-431.

13. Hwang K, Kim Y J, Park H Chung I H. Selective Neurectomy of the Masseteric Nerve in Masseter Hypertrophy. J Craniofac Surg 2004; 15: 780-784. 